\title{
A High-Yield, One-Step Synthesis of o-Phenylene Ethynylene Cyclic Trimer via Precipitation-Driven Alkyne Metathesis
}

\author{
Wei Zhang, Scott M. Brombosz, Jose L. Mendoza and Jeffrey S. Moore* \\ Roger Adams Laboratory, Departments of Chemistry and Materials Science \& \\ Engineering, University of Illinois at Urbana-Champaign, Urbana, Illinois 61801
}

\section{$\underline{\text { Table of Contents }}$}

General Experimental Procedure

pg S2

Characterization data for compound $\mathbf{3}$ and $\mathbf{4}$

pg S3

${ }^{1} \mathrm{H}$ NMR and ${ }^{13} \mathrm{C}$ NMR spectra for compound 5

pg S4

Characterization data for precursors of compound 6

pg S5

References

pg S6 


\section{Supporting Information}

General Procedures. Unless otherwise indicated, all starting materials were obtained from commercial suppliers and were used without further purification. All air or moisture-sensitive manipulations were performed under an atmosphere of argon using standard Schlenk techniques or in an argon filled glove box. $\mathrm{CCl}_{4}$ was distilled over $\mathrm{P}_{2} \mathrm{O}_{5}$ and degassed before use. THF was distilled over Na before use. Analytical thin-layer chromatography (TLC) was performed on precoated silica gel plates. Visualization was performed with UV light $(254 \mathrm{~nm})$ or iodine stain. Flash column chromatography on all monomers and monomer precursors was conducted with silica gel 60 (230-400 mesh, 60 $\AA$ ). Flash column chromatography for macrocycles was conducted with silica gel 62 (60200 mesh, $150 \AA)$.

The ${ }^{1} \mathrm{H}$ and ${ }^{13} \mathrm{C}$ NMR spectra were recorded on 400 or $500 \mathrm{MHz}$ spectrometers. Proton chemical shifts are expressed in parts per million $(\delta)$ using the residual solvent protons as an internal standard. Carbon-13 chemical shifts are also expressed in parts per million $(\delta)$ using the solvent's ${ }^{13} \mathrm{C}$ resonance as an internal standard. Coupling constants $(J)$ are reported in Hertz $(\mathrm{Hz})$, and splitting patterns are designated as s (singlet), d (doublet), $\mathrm{t}$ (triplet), $\mathrm{q}$ (quartet), $\mathrm{m}$ (multiplet) and br (broad). Gel permeation chromatography (GPC) measurements were performed in $\mathrm{THF}$ at $25{ }^{\circ} \mathrm{C}$. The light scattering, mass, and viscosity constants were determined from a single $96 \mathrm{kDa}$ narrow polystyrene standard and checked for accuracy against other known polystyrene standards. The column exclusion limit was $1.0 \cdot 10^{7} \mathrm{Da}$, and the flow rate was 1.0 $\mathrm{mL} / \mathrm{min}$. 
1,2-Diethynyl-4,5-dihexyloxybenzene (3) ${ }^{1}:{ }^{1} \mathrm{H}$ NMR $\left(\mathrm{CDCl}_{3}, 500 \mathrm{MHz}\right): \delta 6.95(\mathrm{~s}, 2 \mathrm{H})$, $3.98(\mathrm{t}, J=7.7 \mathrm{~Hz}, 4 \mathrm{H}), 3.25(\mathrm{~s}, 2 \mathrm{H}), 1.81(\mathrm{~m}, 4 \mathrm{H}), 1.30-1.50(\mathrm{~m}, 12 \mathrm{H}), 0.90(\mathrm{t}, J=6.6$ $\mathrm{Hz}, 6 \mathrm{H}) ;{ }^{13} \mathrm{C} \mathrm{NMR}\left(\mathrm{CDCl}_{3}, 125 \mathrm{MHz}\right): \delta 149.4,117.7,116.4,82.2,79.4,69.1,31.5,28.9$, 25.6, 22.5, 14.0; MS (EI): m/z (\%): 326.2 (38), 242.1 (13), 158.0 (100), 129.0 (8); HRMS $\left(\mathrm{C}_{22} \mathrm{H}_{30} \mathrm{O}_{2}\right)$ : calcd 326.2246, found 326.2245; TLC $\mathrm{R}_{f}=0.12$ (n-hexane).

1,2-Bis(benzoylbiphenyl)ethynyl-4,5-dihexyloxybenzene (4): Application of Sonogashira's general procedure for alkyne coupling ${ }^{2}$ [1,2-dihexyloxy-4,5diethynylbenzene 3 (1.30 g, $3.98 \mathrm{mmol})$, 4-benzoyl-4'-bromobiphenyl (2.82 g, 8.36 mmol), $\mathrm{Pd}_{2}(\mathrm{dba})_{3}(0.22 \mathrm{~g}, 0.24 \mathrm{mmol}), \mathrm{CuI}(0.015 \mathrm{~g}, 0.079 \mathrm{mmol}), \mathrm{PPh}_{3}(0.42 \mathrm{~g}, 1.6$ mmol) in triethylamine $(10 \mathrm{~mL})$ and $\operatorname{DMF}(20 \mathrm{~mL})]$ at $65{ }^{\circ} \mathrm{C}$ gave the product as a light yellow solid (2.80 g, 84\%). ${ }^{1} \mathrm{H}$ NMR ( $\left.\mathrm{CDCl}_{3}, 400 \mathrm{MHz}\right): \delta 7.90(\mathrm{~d}, J=8.0 \mathrm{~Hz}, 4 \mathrm{H}), 7.84$ (m, 4H), $7.73(\mathrm{~d}, J=8.1 \mathrm{~Hz}, 4 \mathrm{H}), 7.58-7.70(\mathrm{~m}, 10 \mathrm{H}), 7.51(\mathrm{~m}, 4 \mathrm{H}), 7.07(\mathrm{~s}, 2 \mathrm{H}), 4.06(\mathrm{t}$, $J=7.6 \mathrm{~Hz}, 4 \mathrm{H}), 1.85(\mathrm{~m}, 4 \mathrm{H}), 1.30-1.54(\mathrm{~m}, 12 \mathrm{H}), 0.91(\mathrm{t}, J=6.6 \mathrm{~Hz}, 6 \mathrm{H}) ;{ }^{13} \mathrm{C} \mathrm{NMR}$ $\left(\mathrm{CDCl}_{3}, 100 \mathrm{MHz}\right): \delta 196.2,149.4,144.2,139.5,137.6,136.5,132.5,132.0,130.8$, $130.0,128.3,127.2,126.8,123.5,118.5,115.7,91.8,90.0,69.2,31.5,29.0,25.6,22.6$, 14.0; MS (FAB): $[\mathrm{M}]+$ calcd 839.4, found 839.4; HR-MS $\left(\mathrm{C}_{60} \mathrm{H}_{54} \mathrm{O}_{4}\right)$ : calcd 839.4100, found 839.4100; TLC $\mathrm{R}_{f}=0.25$ (n-hexane/ $\mathrm{CH}_{2} \mathrm{Cl}_{2}, 3 / 2$, v/v); Anal. Calcd for $\mathrm{C}_{60} \mathrm{H}_{54} \mathrm{O}_{4}$ (839.41): C, 85.89; H, 6.49; Found: C, 85.76; H, 6.37. 


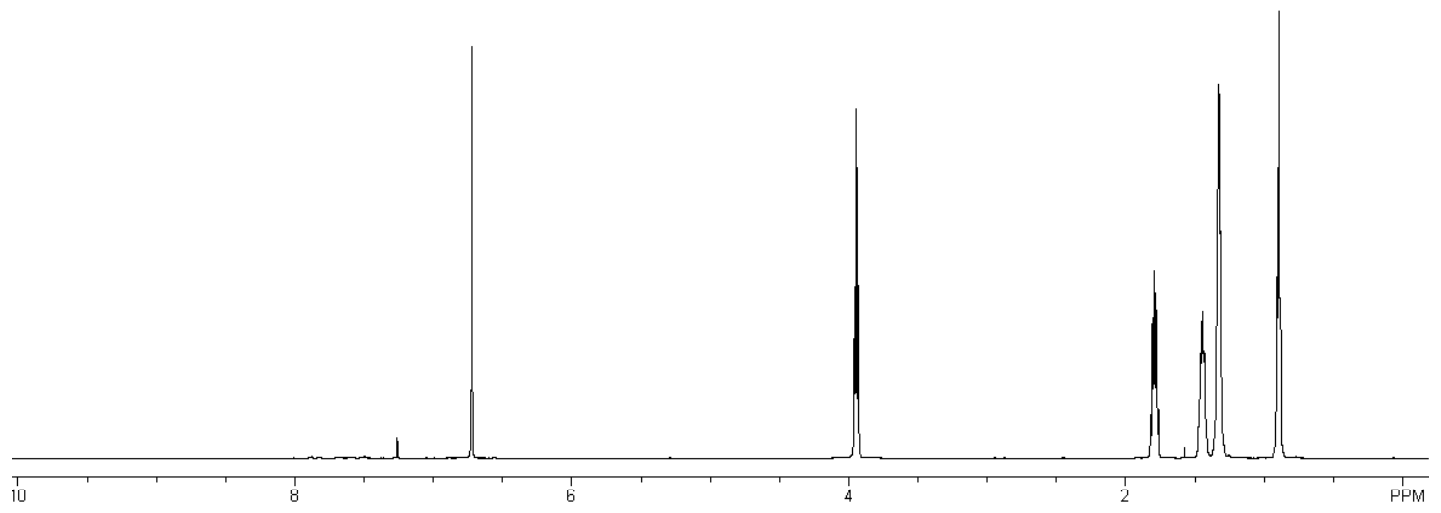

Figure 1. ${ }^{1} \mathrm{H}$ NMR spectrum of cyclic trimer $5\left(\mathrm{CDCl}_{3}, 400 \mathrm{MHz}, 20^{\circ} \mathrm{C}\right)$

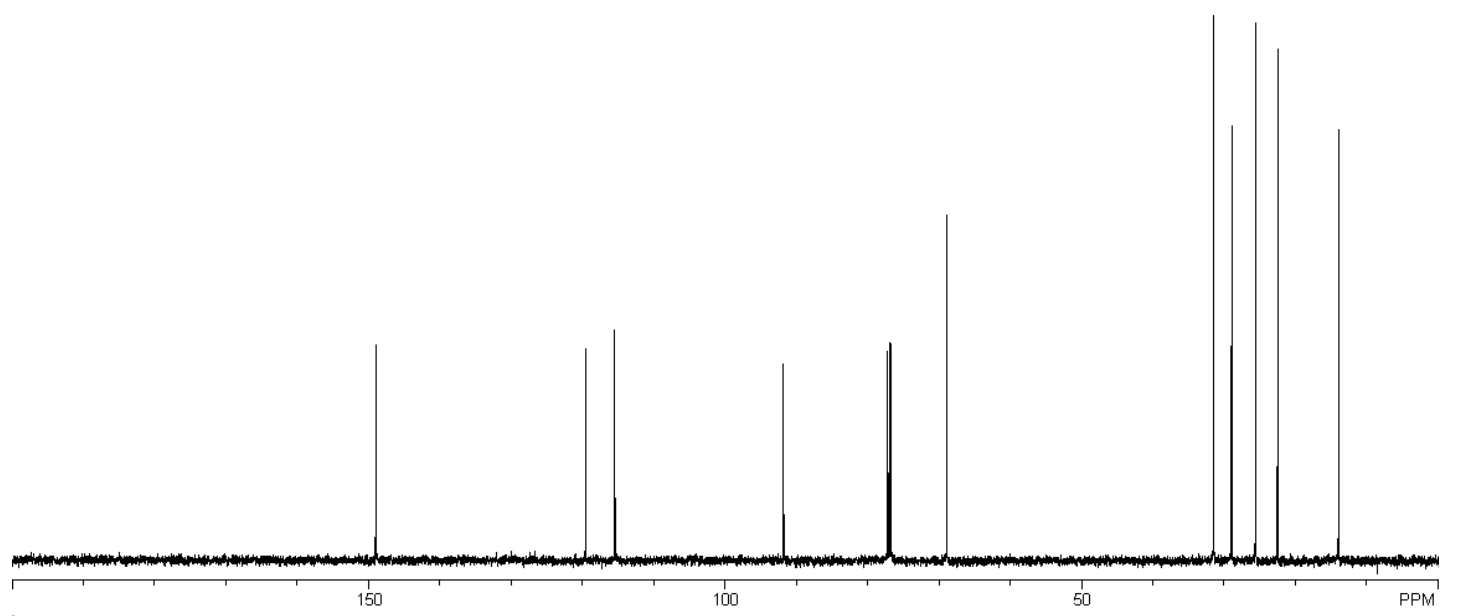

Figure $2 .{ }^{13} \mathrm{C}$ NMR spectrum of cyclic trimer $5\left(\mathrm{CDCl}_{3}, 100 \mathrm{MHz}, 20^{\circ} \mathrm{C}\right)$ 

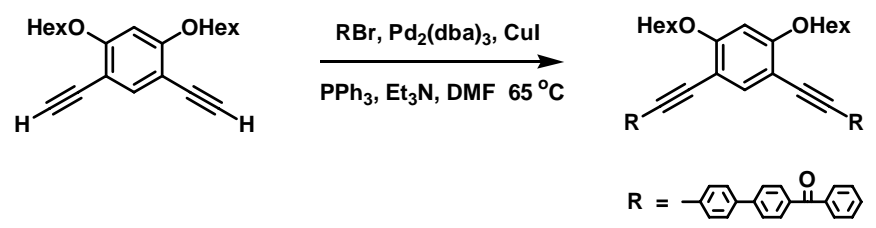

1,3-Diethynyl-4,6-dihexyloxybenzene ${ }^{1}:{ }^{1} \mathrm{H}$ NMR $\left(\mathrm{CDCl}_{3}, 500 \mathrm{MHz}\right): \delta 7.52(\mathrm{~s}, 1 \mathrm{H})$, $6.36(\mathrm{~s}, 1 \mathrm{H}), 4.02(\mathrm{t}, J=7.6 \mathrm{~Hz}, 4 \mathrm{H}), 3.16(\mathrm{~s}, 2 \mathrm{H}), 1.84(\mathrm{~m}, 4 \mathrm{H}), 1.30-1.52(\mathrm{~m}, 12 \mathrm{H})$, $0.90(\mathrm{t}, J=6.8 \mathrm{~Hz}, 6 \mathrm{H}) ;{ }^{13} \mathrm{C} \mathrm{NMR}\left(\mathrm{CDCl}_{3}, 125 \mathrm{MHz}\right): \delta 161.9,139.0,103.8,96.8,79.8$, 79.1, 69.0, 31.5, 28.9, 25.5, 22.5, 14.0; MS (EI): m/z (\%): 326.2 (54), 242.1 (11), 158.0 (100), 138.1 (8), 102.0 (10); HR-MS $\left(\mathrm{C}_{22} \mathrm{H}_{30} \mathrm{O}_{2}\right)$ : calcd 326.2246, found 326.2251; TLC $\mathrm{R}_{f}=0.17\left(n\right.$-hexane $\left./ \mathrm{CH}_{2} \mathrm{Cl}_{2}, 4 / 1, \mathrm{v} / \mathrm{v}\right)$.

1,3-Bis(benzoylbiphenyl)ethynyl-4,6-dihexyloxybenzene: Application of Sonogashira's general procedure for alkyne coupling ${ }^{2}$ [1,3-diethynyl-4,6dihexyloxybenzene (2.07 g, $6.35 \mathrm{mmol}$ ), 4-benzoyl-4'-bromobiphenyl (4.49 g, 13.3 mmol), $\mathrm{Pd}_{2}(\mathrm{dba})_{3}(0.35 \mathrm{~g}, 0.38 \mathrm{mmol}), \mathrm{CuI}(0.012 \mathrm{~g}, 0.064 \mathrm{mmol}), \mathrm{PPh}_{3}(0.67 \mathrm{~g}, 2.5$ mmol) in triethylamine $(17 \mathrm{~mL})$ and $\operatorname{DMF}(31 \mathrm{~mL})]$ at $65{ }^{\circ} \mathrm{C}$ gave the product as a light yellow solid (4.43 g, 83\%). ${ }^{1} \mathrm{H}$ NMR ( $\left.\mathrm{CDCl}_{3}, 500 \mathrm{MHz}\right): \delta 7.90(\mathrm{~d}, J=7.9 \mathrm{~Hz}, 4 \mathrm{H}), 7.84$ (m, 4H), $7.73(\mathrm{~d}, J=8.0 \mathrm{~Hz}, 4 \mathrm{H}), 7.67(\mathrm{~s}, 1 \mathrm{H}), 7.58-7.66(\mathrm{~m}, 10 \mathrm{H}), 7.51(\mathrm{~m}, 4 \mathrm{H}), 6.46(\mathrm{~s}$, 1H), $4.10(\mathrm{t}, J=7.7 \mathrm{~Hz}, 4 \mathrm{H}), 1.90(\mathrm{~m}, 4 \mathrm{H}), 1.60(\mathrm{~m}, 4 \mathrm{H}), 1.34-1.45(\mathrm{~m}, 8 \mathrm{H}), 0.91(\mathrm{t}, J=$ $6.6 \mathrm{~Hz}, 6 \mathrm{H}) ;{ }^{13} \mathrm{C} \mathrm{NMR}\left(\mathrm{CDCl}_{3}, 125 \mathrm{MHz}\right): \delta 196.3,161.4,144.4,139.1,137.7,137.3$, $136.4,132.4,131.9,130.8,130.0,128.3,127.1,126.8,123.9,105.1,97.0,92.0,86.6$, 69.0, 31.6, 29.1, 25.7, 22.6, 14.0; MS (FAB): m/z (\%): 839.5 (77), 670.3 (14), 427.2 (8), 271.1 (15), 154.1 (68); HR-MS $\left(\mathrm{C}_{60} \mathrm{H}_{54} \mathrm{O}_{4}\right)$ : calcd 839.4100, found 839.4100; TLC $\mathrm{R}_{f}=$ 0.16 (n-hexane/ $\mathrm{CH}_{2} \mathrm{Cl}_{2}, 1 / 1, \mathrm{v} / \mathrm{v}$ ); Anal. Calcd for $\mathrm{C}_{60} \mathrm{H}_{54} \mathrm{O}_{4}$ (839.41): C, 85.89; H, 6.49; Found: C, 85.55; H, 6.48 . 


\section{References:}

1. Synthesis of 3 followed the procedures for preparation of 4,5-dioctyloxy-1,2diethynylbenzene, see (a) Zhou, Q.; Carroll, P. J.; Swager, T. M. J. Org. Chem. 1994, 59, 1294-1301. (b) Bowles, D. M.; Palmer, G. J.; Landis, C. A.; Scott, J. L.; Anthony, J. E. Tetrahedron, 2001, 57, 3753-3760.

2. (a) Sonogashira, K.; Tohda, Y.; Hagihara, N. Tetrahedron Lett. 1975, 4467-4470. (b) Sonogashira, K.; Yatake, T.; Tohda, Y.; Takahashi, S.; Hagihara, N. J. Chem. Soc. Chem. Commun. 1977, 291-292. 